\title{
Correction to: Does mangrove plantation reduce coastal erosion? Assessment from the west coast of India
}

\section{Saudamini Das ${ }^{1}$}

Published online: 3 August 2020

(C) Springer-Verlag GmbH Germany, part of Springer Nature 2020

\section{Correction to: Regional Environmental Change} https://doi.org/10.1007/s10113-020-01637-2

Our recently published paper contained errors in tables 2 and 3. Corrected tables are provided here.

The original article has been corrected.

Publisher's note Springer Nature remains neutral with regard to jurisdictional claims in published maps and institutional affiliations.

The online version of the original article can be found at https://oi.org/ $10.1007 /$ s10113-020-01637-2

Saudamini Das

saudamini@iegindia.org; sdas_28@yahoo.co.in

1 Institute of Economic Growth, University of Delhi Enclave, Room 209, North Campus, Delhi 110007, India 
Table 2 Estimated coefficients of variables on tehsil level erosion in Gujarat

\begin{tabular}{|c|c|c|c|c|c|c|}
\hline \multirow[t]{2}{*}{ Variables } & \multicolumn{2}{|c|}{ Dep var $=s q \mathrm{~km}$ of erosion in a tehsil } & \multicolumn{4}{|c|}{ Dep var $=s q k m$ of erosion in a tehsil $/ L$} \\
\hline & Model 1 & Model 1A & Model 2 & Model 2A & Model 3 & Model 3A \\
\hline Physical vulnerability index & $3.91(0.62)$ & $5.6(0.79)$ & $0.12(0.83)$ & $0.05(0.24)$ & $0.10(0.71)$ & $-0.16(0.70)$ \\
\hline Rate of growth of population & $4.04(0.25)$ & $4.81(0.24)$ & $0.004(0.01)$ & $-0.16(0.32)$ & $0.10(0.18)$ & $-0.01(0.02)$ \\
\hline$\Delta M$ & $0.07(0.85)$ & & $0.001(1.27)$ & & & \\
\hline$L$ & $0.34 * * *(3.02)$ & $0.24(1.51)$ & & & & \\
\hline$\Delta M / L$ & & & & & $0.94(1.02)$ & \\
\hline Kori Creek & $-13.43(0.77)$ & $-26.54 * *(1.89)$ & $-0.27(1.27)$ & $-0.21(1.22)$ & $-0.52(1.21)$ & $-0.78 * * *(2.94)$ \\
\hline Gulf of Kutch & $-19.51 * * *(3.05)$ & $-12.81 * *(2.12)$ & $-0.37 * * *(3.10)$ & $-0.27 * *(2.23)$ & $-0.76(1.74)$ & $-0.42(1.92)$ \\
\hline Gulf of Khambhat & $9.76(0.99)$ & $9.83(0.98)$ & $0.49(0.78)$ & $0.62(0.82)$ & $0.66(0.91)$ & $0.56(1.06)$ \\
\hline South Saurashtra & $-14.96 * *(2.11)$ & $-13.38 * *(2.00)$ & $-0.34 * *(2.44)$ & $-0.33 * *(2.46)$ & $-0.29(1.38)$ & $-0.56^{* *}(2.17)$ \\
\hline Kori Creek $\times \Delta M$ & & $0.35(1.85)$ & & $0.001 * *(2.33)$ & & \\
\hline Gulf of Kutch $\times \Delta M$ & & $0.03(0.97)$ & & $0.0004(0.92)$ & & \\
\hline Saurashtra $\times \Delta M$ & & $1.66(1.27)$ & & $0.001(0.24)$ & & \\
\hline Gulf of Khambhat $\times \Delta M$ & & $-0.68(1.23)$ & & $0.03(0.52)$ & & \\
\hline South Gujarat $\times \Delta M$ & & $0.52(0.60)$ & & $0.02(1.21)$ & & \\
\hline Kori Creek $\times(\Delta M / L)$ & & & & & & $0.79 * * *(12.18)$ \\
\hline Gulf of Kutch $\times(\Delta M / L)$ & & & & & & $0.01(0.10)$ \\
\hline Saurashtra $\times(\Delta M / L)$ & & & & & & $-0.01(0.34)$ \\
\hline Gulf of Khambhat $\times(\Delta M / L)$ & & & & & & $4.35(1.59)$ \\
\hline South Gujarat $\times(\Delta M / L)$ & & & & & & $0.32(0.97)$ \\
\hline Constant & $5.14(0.44)$ & $-5.58(0.45)$ & $0.25(0.86)$ & $0.34(0.92)$ & $0.11(0.31)$ & $0.76(1.66)$ \\
\hline Number of observations & 44 & 44 & 44 & 44 & 44 & 44 \\
\hline $\mathrm{R}$ square & 0.51 & 0.57 & 0.09 & 0.11 & 0.19 & 0.53 \\
\hline F value & $5.67 \mathrm{p}<0.001$ & $1885.79 \mathrm{p}<0.001$ & $6.89 \mathrm{p}<0.001$ & $159.96 \mathrm{p}<0.001$ & $1.87 \mathrm{p}<0.001$ & $24.95 \mathrm{p}<0.001$ \\
\hline Root mean square error & 21.97 & 21.91 & 1.39 & 1.46 & 1.31 & 1.07 \\
\hline
\end{tabular}

Note: $* * *$ and $* *$ imply $1 \%$ and $5 \%$ level of significance, respectively.

$\Delta M=$ Change in mangrove (in sq. $\mathrm{km}$ ); $L=$ Length of coastline (in $\mathrm{km}$ ); $\Delta M / L=$ Change in mangrove per km of coastline; South Gujarat is the comparison regional dummy. 
Table 3 Estimated coefficients of variables on tehsil level accretion in Gujarat

\begin{tabular}{|c|c|c|c|c|c|c|}
\hline \multirow{2}{*}{$\begin{array}{l}\text { Explanatory variables used in } \\
\text { estimation }\end{array}$} & \multicolumn{2}{|c|}{ Dep var $=s q \mathrm{~km}$ of accretion in a tehsil } & \multicolumn{4}{|c|}{ Dep var $=s q k m$ of accretion in a tehsil $/ L$} \\
\hline & Model 1 & Model 1A & Model 2 & Model 2A & Model 3 & Model 3A \\
\hline Physical vulnerability index & $-6.07(1.21)$ & $-3.86(0.61)$ & $-0.005(0.05)$ & $-0.04(0.36)$ & $-0.02(0.18)$ & $-0.08(0.77)$ \\
\hline Rate of growth of population & $-49.78(1.85)$ & $-46.23(1.69)$ & $-0.58(1.57)$ & $-0.73(1.58)$ & $-0.57(1.53)$ & $-0.63(1.63)$ \\
\hline$\Delta M$ & $0.33(0.90)$ & & $0.003(1.09)$ & & & \\
\hline$L$ & $0.84 * * *(3.67)$ & $0.39 * * *(3.80)$ & & & & \\
\hline$\Delta M / L$ & & & & & $0.41(1.26)$ & \\
\hline Kori Creek & $-6.69(0.15)$ & $-77.65 * * *(5.42)$ & $0.13(0.37)$ & $-0.07(0.46)$ & $0.27(0.88)$ & $-0.76 * *(2.21)$ \\
\hline Gulf of Kutch & $5.59(0.31)$ & $33.20(1.64)$ & $0.33(1.28)$ & $0.47(1.48)$ & $0.27(1.15)$ & $0.39(1.40)$ \\
\hline South Saurashtra & $-11.21(1.01)$ & $-5.94(0.75)$ & $-0.11(1.02)$ & $-0.05(0.53)$ & $-0.09(0.95)$ & $-0.09(0.79)$ \\
\hline Gulf of Khambhat & $11.74(1.33)$ & $14.34 * *(2.07)$ & $0.23(1.30)$ & $0.32(1.50)$ & $0.29(1.63)$ & $0.30 * *(2.03)$ \\
\hline Kori Creek $\times \Delta M$ & & $1.59 * * *(12.12)$ & & $0.005 * * *(11.41)$ & & \\
\hline Gulf of Kutch $\times \Delta M$ & & $0.08(0.38)$ & & $0.002(0.53)$ & & \\
\hline Saurashtra $\times \Delta M$ & & $2.90(1.99)$ & & $0.006(0.33)$ & & \\
\hline Gulf of Khambhat $\times \Delta M$ & & $-0.18(0.72)$ & & $0.007(0.52)$ & & \\
\hline South Gujarat $\times \Delta M$ & & $1.40(1.92)$ & & $0.03 * *(2.08)$ & & \\
\hline Kori Creek $\times(\Delta M / L)$ & & & & & & $2.11 * *(2.54)$ \\
\hline Gulf of Kutch $\times(\Delta M / L)$ & & & & & & $0.18(0.59)$ \\
\hline Saurashtra $\times(\Delta M / L)$ & & & & & & $-0.09(1.19)$ \\
\hline Gulf of Khambhat $\times(\Delta M / L)$ & & & & & & $1.12(1.82)$ \\
\hline South Gujarat $\times(\Delta M / L)$ & & & & & & $0.43 * *(2.68)$ \\
\hline Constant & $-12.82(0.98)$ & $-2.65(0.25)$ & $0.30(1.55)$ & $0.30(1.47)$ & $0.25(1.50)$ & $0.37(1.88)$ \\
\hline Number of observations & 44 & 44 & 44 & 44 & 44 & 44 \\
\hline $\mathrm{R}$ square & 0.71 & 0.85 & 0.20 & 0.22 & 0.26 & 0.38 \\
\hline F value & $4.11 \mathrm{p}<0.001$ & $4930.21 \mathrm{p}<0.001$ & $3.66 \mathrm{p}<0.001$ & $185.98 \mathrm{p}<0.001$ & $2.54 \mathrm{p}<0.001$ & $2.97 \mathrm{p}<0.001$ \\
\hline Root mean square error & 36.74 & 27.98 & 0.54 & 0.56 & 0.51 & 0.50 \\
\hline
\end{tabular}

Note: $* * *$ and $* *$ imply $1 \%$ and $5 \%$ level of significance, respectively.

$\Delta M=$ Change in mangrove (in sq. $\mathrm{km}$ ); $L=$ Length of coastline (in $\mathrm{km}$ ); $\Delta M / L=$ Change in mangrove per km of coastline; South Gujarat is the comparison regional dummy. 